\title{
Image Contrast Improvement in Image Fusion between CT and MRI images of Brain Cancer Patients
}

\author{
Annisa Tenri Maya ${ }^{* 1}$, Suryono Suryono ${ }^{1}$, Choirul Anam ${ }^{1}$ \\ ${ }^{* 1}$ Departement of Physics, Faculty of Science and Mathematics, Diponegoro University, Semarang, Indonesia \\ annisatenri.2017@fisika.fsm.undip.ac.id

\section{ABSTRACT}

\author{
Article Info \\ Volume 8, Issue 1 \\ Page Number: 104-109
}

Publication Issue :
January-February-2021

Article History

Accepted : 10 Jan 2021

Published : 25 Jan 2021
Medical image fusion has been carried out to obtain information benefits from multi-modalities of medical images. The purpose of this study is to improve the image contrast of fusion image with adaptive method. The median filter was implemented to the images before registration to remove noise for obtaining good image fusion. Geometric transformation-based image registration was used to automatically align two images of computed tomography (CT) scanner and magnetic resonance imaging (MRI) to a common coordinate system. After that, the image contrast was improved with adaptive method. Finally, the fused image was assessed using the signal-to-noise ratio (SNR) and contrast-to-noise ratio (CNR). From this study, it was found that the average SNR value in the image fusion before contrast improvement is 0.09 and after that is 0.73 . While the average CNR value in image fusion before contrast improvement is 1.54 and after that is 1.79 . It means that the CNR increases $14.02 \%$.

Keywords : Image fusion, dice coefficient, SNR, CNR

\section{INTRODUCTION}

Brain cancer is one of the most common malignant tumors in the world and has an aggressive character, covering about $2.4 \%$ of cases of malignancy. Brain cancer occurs due to abnormal growth of brain cells and can spread to other areas of the brain or body. Malignant brain cancer is life threatening because of its location in the brain [1]. Treatment of brain cancer is usually performed with radiotherapy because it may not be operable. Radiotherapy has been reported having a high cure rate for some cancerous tissue because it is able to reduce the size and eliminate the cancer [2].
In recent years, external radiotherapy, such as intensity-modulated radiation therapy (IMRT) and 3D conformal radiotherapy (CRT) have been used in many institutions.

The success of radiotherapy is strongly influenced by the accuracy of determining the target volume. Therefore, determining the target volume is the most important part of the radiotherapy treatment planning system (TPS) process. In some cases, it is difficult to determine the exact location of the target due to the lack of information from the image obtained from single modality so that additional imaging from different modalities is required. Therefore, it is necessary to do a fusion technique that allows to combine images from several modalities [3].

Copyright: (C) the author(s), publisher and licensee Technoscience Academy. This is an open-access article distributed under the terms of the Creative Commons Attribution Non-Commercial License, which permits unrestricted noncommercial use, distribution, and reproduction in any medium, provided the original work is properly cited 
Merging multiple images from one or more imaging modalities is called medical image fusion. The main purpose of medical image fusion is to automatically transfer and combine the useful information files contained in multiple image sources to a single unified image without any loss of information. This technique enhances the clinical visualization of medical images for more accurate diagnosis. Medical image fusion is based on the fact that each imaging modality provides limited information [4]. Each modality has different characteristics, such as the image from a computed tomography scanner (CT-scan) provides anatomical or structural information at high spatial resolution [5]. Meanwhile, magnetic resonance imaging (MRI) images can be used to diagnose brain tumors because they provide good information of soft tissue [6]. Accuracy of image fusion is challenging because it integrates images from various formats. With image fusion, radiation oncologists can get effective and accurate information from single image [7]. However, contrast of each original image is very different because it comes from different modalities. Therefore, the contrast should be adjusted to make a fused image showing more effective view and displaying useful information in the image. In this study, we improve the image contrast of fusion image with adaptive method. To assess an effectiveness the proposed approach, image quality is measured using the metrics of signal-to-noise ratio (SNR) and contrast-to-noise ratio (CNR).

\section{METHODS AND MATERIAL}

In this study, images of CT and MRI of brain cancer of five patients who received 3D conformal radiotherapy were collected. The digital images of the brain resulting from the acquisition of CT and MRI modalities were used as an input that will be processed through computer programming. The Matlab R2014b was used.
The steps were carried out into five stages. First, the stage of opening images. Second, the stage of image pre-processing. Third, the application stage of the fusion method. Fourth, the stage of enhancing image quality using the adaptive method. Fifth, the image characterization. The flow chart of image fusion is shown in Figure 1.

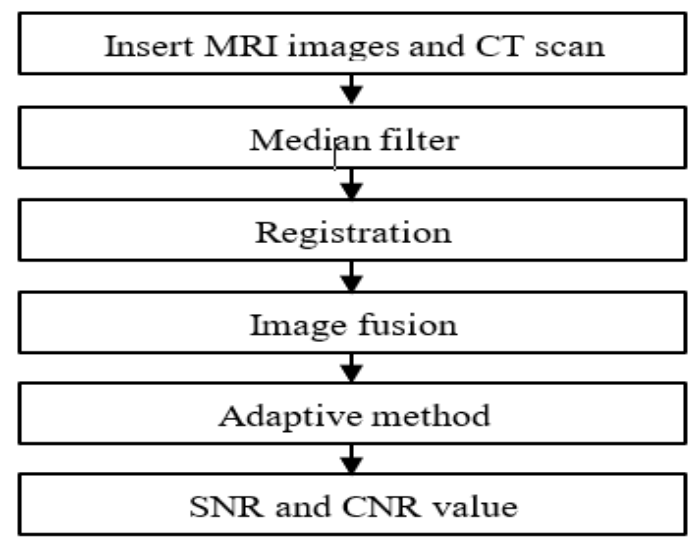

Fig 1. Flow chart of the proposed medical image fusion

The first step was reading each CT and MRI images in the digital imaging and communications in medicine (DICOM) format in the MATLAB program. The process of the area calculation was performed by counting the number of pixels that compose the object in the binary image. After that, CT and MRI images from one patient and having the same image size become the input image. The example of CT and MRI images used are shown in Figure 2.

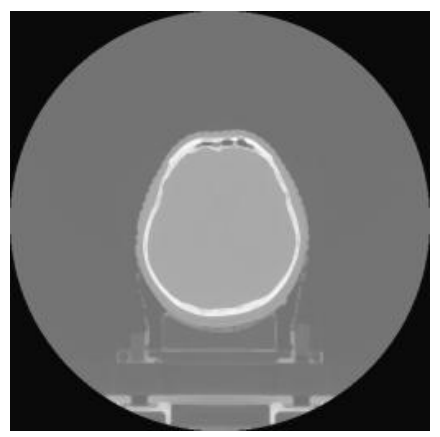

(a)

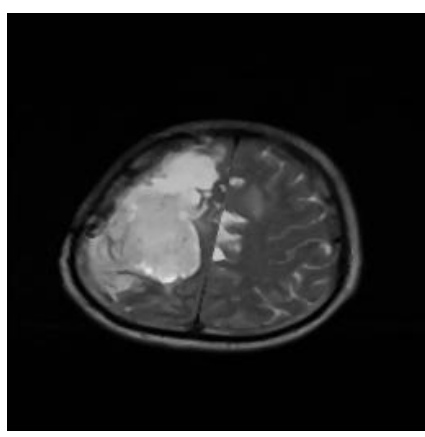

(b)
Fig 2. Input of images for image fusion, (a) CT image and (b) MRI image. 
The second stage was image pre-processing, including image filtering and registration. The input images must be pre-processed. The first pre-processing was filtering images using a median filter to remove noise in the image [4]. The median filter has the same function as the low pass filter, which is to smooth and reduce noise in the image. The process was by sorting the pixel values of a group of pixels within a window, determining the median value of the pixel, and by replacing the processed pixel value with a median value [8]. Figure 3 shows the filtered CT and MRI images.

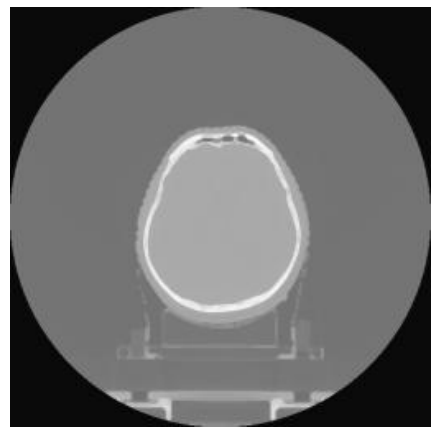

(a)

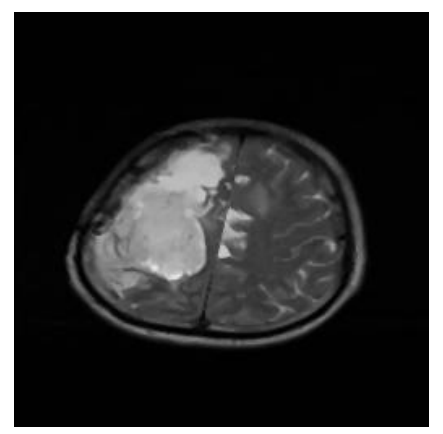

(b)
Fig 3. Filtered images, (a) CT and (b) MRI.

In addition, image registration was also performed as shown in Figure 4 (a). Image registration is the process of determining geometric transformations that connect identical points in two image series: a moving dataset and a stationary source dataset [9]. Image registration is required when integrating multimodality images (e.g. CT, MRI, USG, PET, or SPECT) into treatment planning [10]. The image output required from this registration was a geometric moving image equal to a stationary image.

The complexity of multi-modality image registration depended on the complexity of anatomical variations, including motion and anatomical changes, between imaging or acquisition sessions [6]. The complexity can be reduced if different modalities are not used by maintaining the same patient configuration between imaging sessions, i.e. by using the same immobilization device, minimizing the time interval between image acquisitions to reduce physiological movement. So that it can use a simple image registration technique (rigid registration). Image registration algorithms consisted of metric transformations and geometric transformations. The metric transformation was commonly used based on voxel intensity and features. Geometry transformation was a commonly used image registration transformation algorithm. There were three geometric transformations, namely rigid registration, affine registration, and deformation registration [11].

An affine registration was a registration that includes a transformation from a rigid registration and adds additional transformations of plane scaling, shear, and reflection [12]. As in rigid registrations, the distance between points was maintained but with the addition of parallel lines which remain after being transformed. The result of the affine registration is shown in Figure 4 (b).

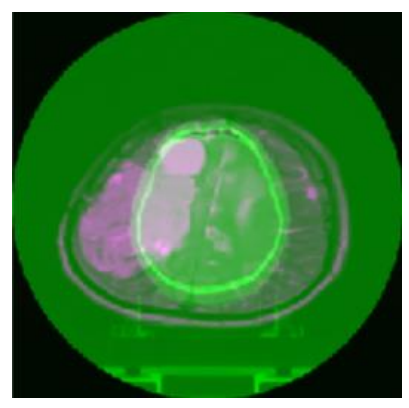

(a)

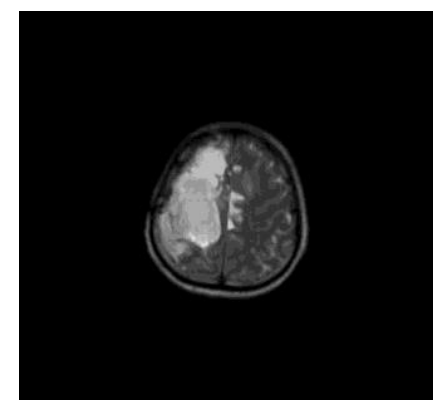

(b)
Fig 4. (a) Fusion image without registration process; and (b) Fusion image with registration process.

The third stage was to combine CT and MRI images using the fusion algorithm. In this research, pixel level image fusion was used. It means that a fusion process that is carried out on the information contained in the registered input image pixels.

The fourth research stage was to improve image contrast using the adaptive method in the Matlab program. This process used intensity adjustment of the fused image that is manually adjusted by changing the intensity value to increase the quality of the image 
[13]. The commands used for the contrast enhancement process are:

\section{$J=$ imadjust_(I,_[low_in high_in],_[low_out high_out])}

Imadjust was a function in Matlab that maps image intensity values so that $1 \%$ of data is saturated at low and high intensities. This process was carried out by changing the intensity of the fused image $(I)$ to the intensity of the output image $(J)$ by mapping the intensity value between "low" and "high" to the intensity value between "bottom" and "top". Intensity values below "low" and above "high" will be truncated, i.e., values below the "low" map downward, and values above the "high" map upwards.

The fifth step was image characterization. The fused image quality was characterized using metrics of the signal-to-noise ratio (SNR) and contrast-to-noise ratio (CNR).

The SNR described the level of difference between the measured signal and the noise that is also included in the measurement results [13]. The greater the SNR value, the easier the signal and noise will be distinguished. SNR can be calculated by dividing the signal height (Is) by the standard value of the the signal $(\sigma)$ as in the equation below [14]:

$$
\mathrm{SNR}=\frac{I s}{\sigma}
$$

High SNR values indicated good performance in fusion and denoising image.

Contrast was a measure of how much the signal can be distinguished from the background. The greater the contrast, the easier the signal will be to distinguish from the background. In contrast to SNR, the CNR was the ratio value between the distance of the signal from the background and the noise in the background area. CNR can be calculated from the signal height (Is), the background height (Ib) and the standard

balance value of the background area $(\sigma)$ as follows [2]:

$$
\mathrm{CNR}=\frac{I s-I b}{\sigma}
$$

\section{RESULTS AND DISCUSSION}

Image fusion was performed on five images after the registration process. Image fusion was performed using edge preservation fusion algorithm so as to smooth the input image and retain edge information from the image. In the fusion process, the registered image was used as a moving image, while the CT scan image was used as a background image in the image fusion. The results of the image fusion for each patient are shown in Figure 6. After that, the adaptive image input process was carried out. This process was to increase the contrast of the fusion image by changing the intensity value. The results of fusion image processing using the adaptive method are shown in Figure 7.

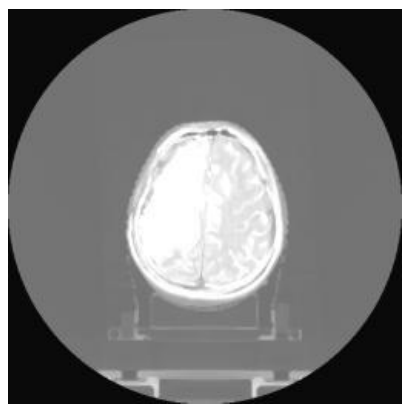

(a)

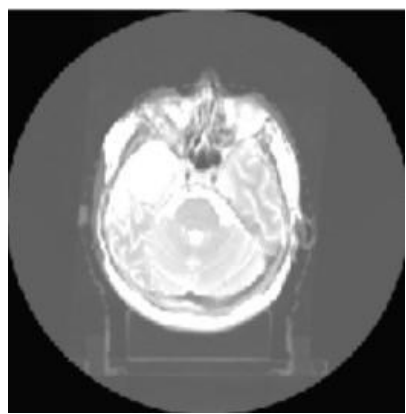

(c)

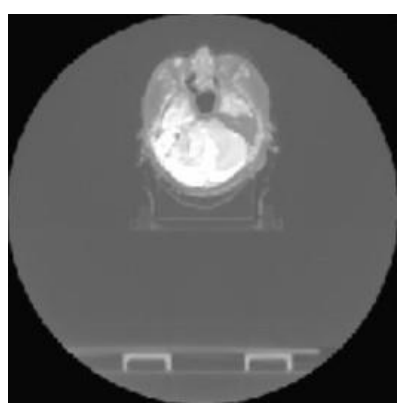

(b)

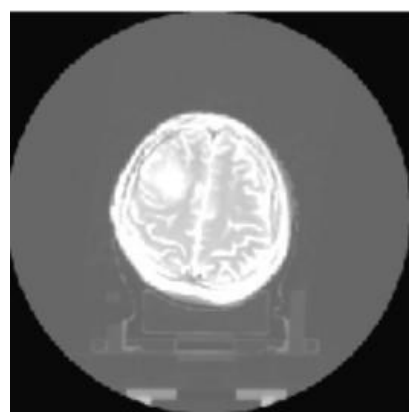

(d) 


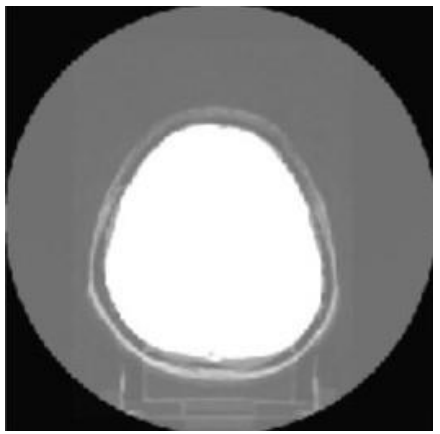

(e)

Fig 6. Images of fusion without adaptive method; (a) first image, (b) second image, (c) third image, (d) fourth image, and (e) fifth image.

To evaluate the performance of image fusion, subjective and objective measurements are used. Subjective evaluation is concerned with visual perception. The objective analysis of image fusion is carried out using the SNR and CNR.

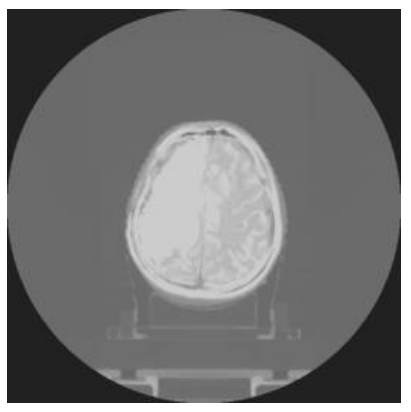

(a)

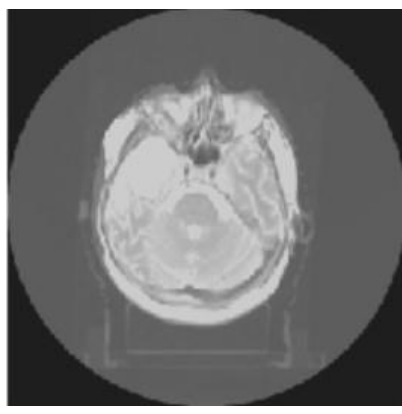

(c)

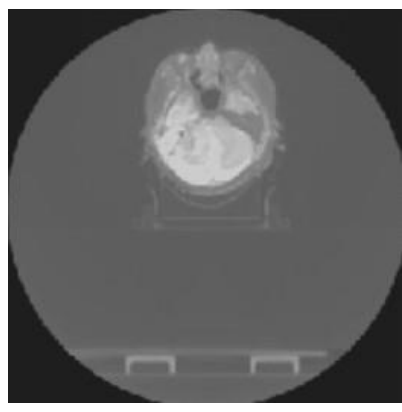

(b)

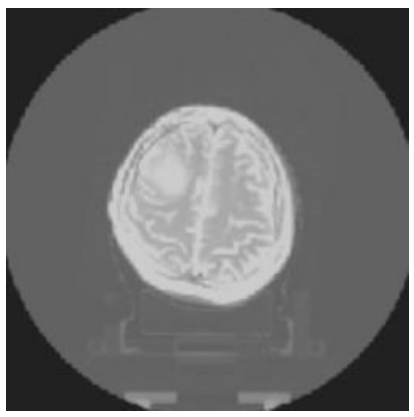

(d)

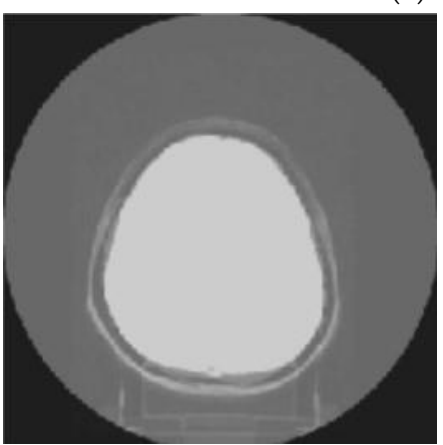

(e)
Fig 7. Image fusion using adaptive method; (a) first image, (b) second image, (c) third image, (d) fourth image, and (e) fifth image

The results of SNR and CNR calculations for each image are shown in Table 1. In the first image shows the percentage increase in SNR of 98.91\% and CNR of $11.80 \%$, the second image shows the percentage of increase in SNR of $98.42 \%$ and CNR of $13.92 \%$, the third image shows the percentage of SNR increase of $98.81 \%$ and CNR of $16.84 \%$, the fourth image shows the percentage of increase in SNR of $98.48 \%$ and CNR of $11.83 \%$, and the fifth image shows the percentage of increase in SNR of $98.40 \%$ and CNR of $15.76 \%$ and with an average percentage increase in SNR of $98.80 \%$ and CNR of $14.03 \%$.

\section{TABLE I}

SNR and CNR of fusion images with and without filtering.

\begin{tabular}{|c|c|c|c|c|c|c|}
\hline \multirow{2}{*}{ Patient } & \multicolumn{2}{|c|}{$\begin{array}{c}\text { Without } \\
\text { contrast } \\
\text { improvement }\end{array}$} & \multicolumn{2}{c|}{$\begin{array}{c}\text { With contrast } \\
\text { improvement }\end{array}$} & \multicolumn{2}{c|}{$\begin{array}{c}\text { Enhancement } \\
(\%)\end{array}$} \\
\cline { 2 - 7 } & SNR & CNR & SNR & CNR & SNR & CNR \\
\hline First & 0.01 & 1.61 & 0.59 & 1.83 & 98.91 & 11.80 \\
\hline Second & 0.01 & 1.65 & 0.81 & 1.92 & 98.42 & 13.92 \\
\hline Third & 0.01 & 1.40 & 0.86 & 1.69 & 98.81 & 16.84 \\
\hline Fourth & 0.01 & 1.51 & 0.59 & 1.71 & 98.48 & 11.83 \\
\hline Fifth & 0.00 & 1.51 & 0.80 & 1.79 & 99.40 & 15.76 \\
\hline Average & 0.09 & 1.54 & 0.73 & 1.79 & 98.80 & 14.03 \\
\hline
\end{tabular}

\section{CONCLUSION}

In this research, image fusion using an intensity-based method and the use of a median filter to remove noise has been carried out. The images used are CT and MRI images with $256 \times 256$ pixels. The MRI image which has the functional image is used as the front ground image and the CT image which has the anatomical image is used as the background image. In this study, it was found that the average SNR value of image fusion before and after contrast enhancement increased by $98.08 \%$. Meanwhile, the average CNR value in the fusion image before and after contrast enhancement increased by $14.04 \%$. From these results, 
it is evident that the contrast enhancement has greater SNR and CNR values than the image without it.

\section{REFERENCES}

[1] M.L. Anisha, M. Balaji, M. Aju, "Multi-Level Fusion of CT and MRI Brain Images for Classifying Tumor", 2014, in International Journal of Enhanced Research in Management \& Computer Applications, 2014, vol. 3, 8, 34-40.

[2] M.J.R. Figueroa, E.Q. Lee, "Brain Tumors", in The American Journal of Medicine, 2018, 131,8, 874-882.

[3] K.K. Brock, S. Mutic, T.R. McNutt, H. Li, M.L. Kessler, "Use of image registration and fusion algorithms and techniques in radiotherapy", Report of the AAPM Radiation Therapy Committee Task Group No. 132, in Medical Physics, 2017, 44,7, 43-76.

[4] S. Liu, T. Zhang, J. Zhao, H. Li, "Medical image fusion based on nuclear norm minimization", Int Journal Imaging System Technology, 2015, 25,310-316.

[5] C. Anam, T. Fujibuchi, F. Haryanto, W.S. Budi, H. Sutanto, K. Adi, Z. Muhlisin Z, G. Dougherty, "Automated MTF measurement in CT images with a simple wire phantom", Polish Journal of Medical Physics and Engineering. 2019, 25, 179-187

[6] A.J. Pinakas, S. Paul, P. Pushpalatha, "A Comparative Study of Image Fusion in MRI and CT Images", International Journal of Mechanical Engineering and Information Techonolgy, 2014, 2, 11, 875-882.

[7] K.R. Padgett, R. Stoyanova, S. Pirozzi, P. Johnson, J. Piper, N. Dogan, A. Pollack, "Validation of a deformable MRI to CT registration algorithm employing same day planning MRI for surrogate analysis", Journal of Applied Clinical Medical Physics, 2018, 19,2, 258-264.

[8] D.P. Bavirisetti, V. Kollu, X. Gang, R. Dhuli, "Fusion of MRI and CT Images Using Guided Image Filter And Image Statistics", in International Journal Imaging System Technology, 2017, 27, 227-237.

[9] Y. Akayama, N. Kadoya, T. Yamamoto, K. Ito, M. Chiba, K. Fujiwara, K. Jingu, "Evaluation of the performance of deformable image registration between planning CT and CBCT images for the pelvic region: comparison between hybrid and intensity-based DIR", in Journal of Radiation Research, 2017, 58,4, 567-571.

[10] K.R. Padgett, R. Stoyanova, S. Pirozzi, P. Johnson, J. Piper, N. Dogan, A. Pollack, "Validation of a deformable MRI to CT registration algorithm employing same day planning MRI for surrogate analysis", in Journal of Applied Clinical Medical Physics, 2018, 19,2, 258-264.

[11] E. Thomas, B.P. Nair, S. John, M. Dominic, "Image Fusion using Daubechies Complex Wavelet Transform and Lifting Wavelet Transform: A multiresolution approach", in International Conference on Magnetics Machines \& Drives, pp,1-5, 2014.

[12] A.K., Vikrant, B. Himanshi, A. Sahu, "Medical Image Fusion using Combination of PCA and Wavelet Analysis", in International Conference on Advances in Computing Communications and Informatics, pp, 986991, 2014.

[13] L. Zhang, F. Yin, B. Moore, S. Han, J. Cai, "A Multisource Adaptive Magnetic Resonance Image Fusion Technique for Versatile Contrast Magnetic Resonance Imaging”, in Cancer Transl Medical, 2018, 4,3,65-9.

[14] A.A. Ben, P. Ping, "Peak SNR in automated coronary calcium scoring: Selecting CT scan parameters and statistically defined scoring thresholds", in Medical Physics, 2010, Vol. 37, No. 7.

\section{Cite This Article :}

Annisa Tenri Maya, Suryono Suryono, Choirul Anam, " Image Contrast Improvement in Image Fusion between CT and MRI images of Brain Cancer Patients", International Journal of Scientific Research in Science and Technology(IJSRST), Print ISSN : 2395-6011, Online ISSN : 2395-602X,Volume 8, Issue 1, pp.104-110, January-February-2021. Available at doi $\quad:$ https://doi.org/10.32628/IJSRST218110 Journal URL : http://ijsrst.com/IJSRST218110 